1 Hacettepe Journal of Mathematics and Statistics

$\bigcap$ Volume 47 (6) (2018), 1495-1502

\title{
A note on the stability of a neural field model
}

\author{
Berrak Özgür*†, Ali Demir ${ }^{\ddagger}$ and Sertaç Erman ${ }^{\S}$
}

\begin{abstract}
In this paper we consider the neural field model for two neural populations. We investigate the properties of D-curves and we give some conditions for asymptotic stability. The asymptotic stability region is determined by using the Stépán's formula. Taking various delay terms into account, the effect of delay on the stability is investigated. Moreover we study on the stability cases by considering the real roots of the characteristic equation.
\end{abstract}

Keywords: Neural field model, asymptotic stability.

Mathematics Subject Classification (2010): 37C75, 34K20, 45J05

Received : 01.05.2016 Accepted : 23.09.2016 Doi : 10.15672/HJMS.2017.451

\section{Introduction}

The neural field models are one of the very interesting topics in neuroscience. The studies made by Wilson and Cowan, Amari [12],[1] are very important in this area. According to the study [9] by Veltz, neural field equations describe the activity of neural populations at a mesoscopic level. These models are made to describe the mean activity of neural populations. In these models, integro differential equations are used. For some biological cases the time is needed, hence the delay terms are added in these models. The propagation delay terms in [4],[9]-[10] are added to describe the time for a signal to travel a distance. The delay terms may be constant or space dependent.

The scientists are very interested in neural field theory. The stability of this model is considered in many papers. A center manifold result is given by Veltz and Faugeras in [11]. The existence and uniqueness of the solution is studied in [4] by Faye and Faugeras.

*Department of Mathematics, Faculty of Arts and Sciences, Kocaeli University, Umuttepe Campus, 41380, Kocaeli, Turkey, Email: berrak.ozgur@kocaeli.edu.tr

${ }^{\dagger}$ Corresponding Author.

$\ddagger$ Department of Mathematics, Faculty of Arts and Sciences, Kocaeli University, Umuttepe Campus, 41380, Kocaeli, Turkey, Email: ademir@kocaeli.edu.tr

$\S$ Department of Mathematics, Faculty of Arts and Sciences, Kocaeli University, Umuttepe Campus, 41380, Kocaeli, Turkey, Email: 106133002@kocaeli.edu.tr 
The effect of the delay on the stability is studied by Faye, Faugeras and Veltz in [4],[9][10].

The main aim of this study is to investigate the asymptotic stability conditions for a neural field system. In [9], the stability analysis of a linearized neural field equation for one neural population is made. The main focus of this study is to make the stability analysis of the model for two neuron populations. The difference of this work is that the neurons in the first population are in a relation among themselves and also the effect of the neurons in the second population on the ones in the first population is considered. To make the stability analysis we use the D-subdivision method for which we need the characteristic equation of the linearized system. The process of this method is to separate the parameter space in subspaces after finding the D-curves on which pure imaginary roots locate. This method is also used to make the stability analysis of the Hayes equation, the Cushing equation, the delayed Mathieu equation [6]. The stability analysis of a differential equation including a state dependent delay is made by using this method [2]. In this research, we study on the number of characteristic roots with positive real parts in the subspaces of parameter space. We find the asymptotic stability region by using the Stépán's formula in the subsection 2.1. For a special case, by considering the roots of characteristic equation as real roots, we investigate the conditions for the asymptotic stability and we give a theorem in the subsection 2.2.

\section{Stability analysis via D-subdivision method and Stépán's for- mula}

Consider the neural field model for $p$ neural population on the space $\Omega \subset R^{d}$ which presents the dynamics of mean membran potential with periodic boundary conditions

$$
\begin{gathered}
\left(\frac{d}{d t}+l_{i}\right) V_{i}(t, r)=\sum_{j=1}^{p} \int_{\Omega} J_{i j}(r, \bar{r}) S\left[\sigma_{j}\left(V_{j}\left(t-\tau_{i j}(r, \bar{r}), \bar{r}\right)-h_{j}\right)\right] d \bar{r}+I_{i}^{e x t}(r, t) \\
t \geq 0,1 \leq i \leq p \\
V_{i}(t, r)=\phi_{i}(t, r) \quad t \in[-T, 0]
\end{gathered}
$$

given in $[10,11]$.

In this model, $S$ is the sigmoid function. The functions $\phi_{i}(t, r)$ are the initial conditions. The external currents from other cortical areas are indicated by $I_{i}^{e x t}(r, t)$. The threshold activity for each population are indicated by $h_{i}$. Positive real values $\sigma_{i}$ represent the slope of the sigmoids at the origin. Positive real values $l_{i}$ represent the speed of decrease of the membrane potential toward its rest value.

In this study we consider a special case for the linear neural field model for two neural populations. We consider that neurons in the first neuron population excite each other and also the neurons in the second neuron population excite the ones in the first population. But neurons in the second population inhibit each other and the neurons in the first population inhibit the ones in the second population. In a mathematical way we consider the case $J_{21}(x, y)=J_{22}(x, y)=0$.

For this study we consider $x, y \in\left[\frac{-\pi}{2}, \frac{\pi}{2}\right]$. Here we describe synaptic inputs for a large group of neurons at position $x$ and time $t$ by the functions $V_{1}(x, t)$ and $V_{2}(x, t)$ and their time derivatives by $\frac{d}{d t} V_{1}(x, t)$ and $\frac{d}{d t} V_{2}(x, t)$. The functions $V_{1}(y, t-\tau(x-y))$ and $V_{2}(y, t-\tau(x-y))$ give the firing rate of a large group of neurons with delay $\tau$. The synaptic connectivity is given by the function $J_{i j}(x, y)$ which is even and $\pi$ periodic. Here $J_{11}(x, y)$ describe how neurons in the first neural population at position $y$ affects the neurons in the same population at position $x$ and $J_{12}(x, y)$ describe how neurons 
in the second neural population at position $y$ affects the neurons in the first population at position $x$. In [3], need for the linearization based on the study of McCormick [7] is considered. According this, in order to examine the stability of the solutions of this model, we use the linearization of (2.1) about $(0,0)$. We use $U_{1}(x, t)$ and $U_{2}(x, t)$ instead of $V_{1}(x, t)$ and $V_{2}(x, t)$. For the delay term, we assume $\tau(x-y)=\tau$.

Hence the system for this study is the following:

$$
\begin{aligned}
\frac{d}{d t} U_{1}(x, t)+l_{1} U_{1}(x, t)= & \sigma_{1} s_{1} \int_{\frac{-\pi}{2}}^{\frac{\pi}{2}} J_{11}(x, y) U_{1}(y, t-\tau(x-y)) d y \\
& +\sigma_{2} s_{1} \int_{\frac{-\pi}{2}}^{\frac{\pi}{2}} J_{12}(x, y) U_{2}(y, t-\tau(x-y)) d y \\
\frac{d}{d t} U_{2}(x, t)+l_{2} U_{2}(x, t)= & 0
\end{aligned}
$$

where $s_{1}=S_{0}^{\prime}(0)$.

The asymptotic stability analysis of nonlinar system (2.1) is equivalent to that of system (2.2) about $(0,0)$.

To determine the asymptotic stability region of the neural field model we make use of the D-subdivision method. Hence we need the characteristic equation of the liearized model. By using the exponential perturbation [9] we look for the solutions as $U(x, t)=U(x) e^{\lambda t}$, hence we get

$$
\begin{gathered}
\lambda e^{\lambda t} U_{1}(x)+l_{1} e^{\lambda t} U_{1}(x)-\sigma_{1} s_{1} e^{-\lambda \tau} e^{\lambda t} \int_{\frac{-\pi}{2}}^{\frac{\pi}{2}} J_{11}(x, y) U_{1}(y) d y-\sigma_{2} s_{1} e^{-\lambda \tau} e^{\lambda t} \int_{\frac{-\pi}{2}}^{\frac{\pi}{2}} J_{12}(x, y) U_{2}(y) d y=0 \\
\lambda e^{\lambda t} U_{2}(x)+l_{2} e^{\lambda t} U_{2}(x)=0
\end{gathered}
$$

Hence

$$
\begin{aligned}
& \lambda U_{1}(x)+l_{1} U_{1}(x)-\sigma_{1} s_{1} e^{-\lambda \tau} \int_{\frac{-\pi}{2}}^{\frac{\pi}{2}} J_{11}(x, y) U_{1}(y) d y- \\
& -\sigma_{2} s_{1} e^{-\lambda \tau} \int_{\frac{-\pi}{2}}^{\frac{\pi}{2}} J_{12}(x, y) U_{2}(y) d y=0 \\
& \lambda U_{2}(x)+l_{2} U_{2}(x)=0
\end{aligned}
$$

Since the boundary conditions given in the model are periodic then the solutions of this system are functions $\cos (2 n x)$ and $\sin (2 n x)$ [9]. In this model we consider $F_{1}=$ $\int_{\frac{-\pi}{2}}^{\frac{\pi}{2}} J_{11}(y) U_{1}(y) d y$ and $F_{2}=\int_{\frac{-\pi}{2}}^{\frac{\pi}{2}} J_{12}(y) U_{2}(y) d y$.

Hence the equation for the characteristic values $\lambda$ is

$$
\lambda^{2}+\lambda l_{1}+\lambda l_{2}+l_{1} l_{2}-K \lambda e^{-\lambda \tau} F_{1}-K l_{2} e^{-\lambda \tau} F_{1}=0
$$

or

$$
\lambda^{2}+\lambda l_{2}+l_{1}\left(\lambda+l_{2}\right)-K\left(\lambda e^{-\lambda \tau} F_{1}+l_{2} e^{-\lambda \tau} F_{1}\right)=0
$$


2.1. General case : Complex roots. Writing $\lambda=\mu+i \nu$ and splitting the real and imaginary parts of $(2.4)$ we have

$$
\begin{aligned}
& R e: \mu^{2}-\nu^{2}+\mu l_{2}+\mu l_{1}+l_{1} l_{2}-K \mu e^{-\mu \tau} \cos (\tau \nu) F_{1}-K \nu e^{-\mu \tau} \sin (\tau \nu) F_{1} \\
& -K l_{2} e^{-\mu \tau} \cos (\tau \nu) F_{1}=0 \\
& \text { Im }: 2 \mu \nu+\nu l_{2}+\nu l_{1}-K \nu e^{-\mu \tau} \cos (\tau \nu) F_{1}+K \mu e^{-\mu \tau} \sin (\tau \nu) F_{1} \\
& +K l_{2} e^{-\mu \tau} \sin (\tau \nu) F_{1}=0
\end{aligned}
$$

To determine the $\mathrm{D}$-curves we take $\mu=0$ and then we have

$$
\begin{aligned}
& P\left(\nu, l_{1}, K\right)=-\nu^{2}+l_{1} l_{2}-K \nu \sin (\tau \nu) F_{1}-K l_{2} \cos (\tau \nu) F_{1}=0 \\
& R\left(\nu, l_{1}, K\right)=\nu l_{2}+\nu l_{1}-K \nu \cos (\tau \nu) F_{1}+K l_{2} \sin (\tau \nu) F_{1}=0
\end{aligned}
$$

We take the parameter space $\left(l_{1}, K\right)$ and for $\tau \nu \neq n \pi$ and $F_{1} \neq 0$ we have

$$
l_{1}=\frac{-\nu \cos (\tau \nu)}{\sin (\tau \nu)} \text { and } K=\frac{-\nu}{\sin (\tau \nu) F_{1}}
$$

as the boundaries of D-subdivision. Moreover the line $l_{1} l_{2}-K l_{2} F_{1}=0$ for $\nu=0$ is also a boundary of D-subdivision. We call this singular line as $C_{*}\left(F_{1}, l_{2}\right)$.

Now we investigate some properties of D-curves as in [5]. Assuming $J_{n}=(n \pi,(n+$ $1) \pi$ ), for fixed values of $l_{2}$ and $F_{1}$, we show the D-curves by $C_{n}\left(F_{1}, l_{2}\right)$ in the parameter space $\left(l_{1}, K\right)$. In the following lemmas we take $\theta=\tau \nu$.

2.1. Lemma. The curves $C_{n}\left(F_{1}, l_{2}\right)$ don't intersect each other.

Proof. Assume that $l_{1}\left(\theta_{1}\right)=l_{2}\left(\theta_{2}\right)$ and $K_{1}\left(\theta_{1}\right)=K_{2}\left(\theta_{2}\right)$ for $\theta_{1} \in J_{a}$ and $\theta_{2} \in J_{b}, a \neq b$. Hence we have

$$
\frac{-\theta_{1} \cos \left(\theta_{1}\right)}{\tau \sin \left(\theta_{1}\right)}=\frac{-\theta_{2} \cos \left(\theta_{2}\right)}{\tau \sin \left(\theta_{2}\right)} \text { and } \frac{-\theta_{1}}{\tau \sin \left(\theta_{1}\right) F_{1}}=\frac{-\theta_{2}}{\tau \sin \left(\theta_{2}\right) F_{1}} .
$$

Writing the second equality in the first one we have $\cos \left(\theta_{1}\right)=\cos \left(\theta_{2}\right)$. Therefore we get $\theta_{1}=\theta_{2}$. We conclude that our assumption is wrong and the curves $C_{n}\left(F_{1}, l_{2}\right)$ don't intersect each other.

2.2. Lemma. The curves $C_{n}\left(F_{1}, l_{2}\right)$ intersect the line $l_{1}=0$ only once. For the $K$ coordinates $K_{e}$ where the curves $C_{n}\left(F_{1}, l_{2}\right)$ intersect the line $l_{1}=0$ we have

$$
\begin{aligned}
& K_{e}>K_{e_{+1}} \text { for } e \geq 0 \\
& K_{e}<K_{e_{+1}} \text { for } e<0 \\
& \text { where } \begin{array}{c}
e=f, n=2 f, \quad f \in N \\
e=-f, n=2 f-1, \quad f \in N
\end{array} \\
& e=\left\{\begin{array}{l}
e=1,
\end{array}\right.
\end{aligned}
$$

Proof. Assuming $l_{1}=0$ we get $\theta=\frac{\pi}{2}+2 e \pi$. Taking this $\theta$ value we get the $K$-coordinates uniquely as $K(\theta)=\frac{-(\pi+4 e \pi)}{2 \tau F_{1}}$ where the curves $C_{n}\left(F_{1}, l_{2}\right)$ intersect the line $l_{1}=0$. Considering these $K_{e}$-coordinates we get

$$
\begin{aligned}
& K_{e}>K_{e_{+1}} \text { for } e \geq 0 \\
& K_{e}<K_{e_{+1}} \text { for } e<0 .
\end{aligned}
$$


2.3. Lemma. Considering (2.9), the following limits are satisfied:

$$
\begin{aligned}
& \lim _{\theta \rightarrow k \pi^{+}} l_{1}(\theta)=-\infty \\
& \lim _{\theta \rightarrow k \pi^{-}} l_{1}(\theta)=+\infty \\
& \lim _{\theta \rightarrow k \pi^{+}} K(\theta)=+\infty \\
& \lim _{\theta \rightarrow k \pi^{-}} K(\theta)=-\infty \\
& \lim _{\theta \rightarrow 2 k \pi^{+}} K(\theta)=-\infty \\
& \lim _{\theta \rightarrow 2 k \pi^{-}} K(\theta)=+\infty \\
& \lim _{\theta \rightarrow 2 k \pi^{+}} l_{1}(\theta)=-\infty \\
& \lim _{\theta \rightarrow 2 k \pi^{-}} l_{1}(\theta)=+\infty
\end{aligned}
$$

Proof. The proof is clear from (2.9).

2.4. Lemma. The curve $C_{0}\left(F_{1}, l_{2}\right)$ intersects the line $C_{*}\left(F_{1}, l_{2}\right)$ only at the point $\left(\lim _{\theta \rightarrow 0} l_{1}(\theta), \lim _{\theta \rightarrow 0} K(\theta)\right)=\left(\frac{-1}{\tau}, \frac{-1}{\tau F_{1}}\right)$. Moreover the curves $C_{n}\left(F_{1}, l_{2}\right)$ for $n \neq 0$ don't intersect the line $C_{*}\left(F_{1}, l_{2}\right)$ at any point.

Proof. Considering and writing (2.9) in the sigular line $C_{*}\left(F_{1}, l_{2}\right)$, we get $\frac{\theta l_{2}}{\tau \sin \theta}(1-\cos \theta)=0$ and hence $\theta=2 n \pi, n=0,1,2, \ldots$. This is a contradiction to selection of $J_{n}$ regions.

To investigate the stability of the model in terms of the number of characteristic roots with positive real parts we use the Stépán's formula [6],[8]. Consider the functions $P\left(\nu, l_{1}, K\right)$ and $R\left(\nu, l_{1}, K\right)$ on the parameter space $\left(l_{1}, K\right)$ and take a point $B\left(l_{0}, K_{0}\right)$ in one of the subspace of the parameter space.

Let $\omega=\rho_{j}, j=1, \ldots, s$ and $\rho_{1} \geq \ldots \geq \rho_{s}$ be the positive real roots of $P\left(\nu, l_{0}, K_{0}\right)$ and let $\omega=\sigma_{i}, i=1, \ldots, s$ and $\sigma_{1} \geq \ldots \geq \sigma_{s}=0$ be the nonnegative real roots of $R\left(\nu, l_{0}, K_{0}\right)$.

If the dimension of (2.4) is even $\left(d=2 m, m \in Z^{+}\right)$then we calculate the number of characteristic roots with positive real parts in this subspace by the formula

$$
k=m+(-1)^{m} \sum_{j=1}^{s}(-1)^{j+1} \operatorname{sgn}\left(R\left(\rho_{j}, l_{0}, K_{0}\right)\right)
$$

If the dimension of (2.4) is odd $\left(d=2 m+1, m \in Z^{+}\right)$then we calculate the number of characteristic roots with positive real parts in this subspace by the formula

$$
k=m+\frac{1}{2}+(-1)^{m}\left[\frac{1}{2}(-1)^{s} \operatorname{sgn}\left(P\left(0, l_{0}, K_{0}\right)+\sum_{j=1}^{s-1}(-1)^{j} \operatorname{sgn}\left(P\left(\sigma_{i}, l_{0}, K_{0}\right)\right)\right]\right.
$$

Now we use the $\nu$-parametric expressions in (2.9) to draw the D curves in the parameter space $\left(l_{1}, K\right)$. The asymptotic stability region (where $k=0$ ) and the number of unstable characteristic roots in the subregions of the parameter space is shown in the following graphs. Also the effect of the delay term on the stability is shown below. 


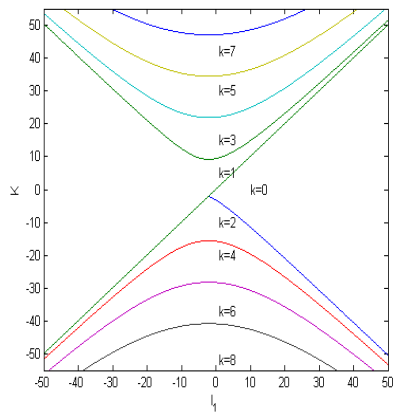

Figure 1. Stability region for the system (2) where $\tau=0.5$ and $F_{1}=l_{2}=1$.

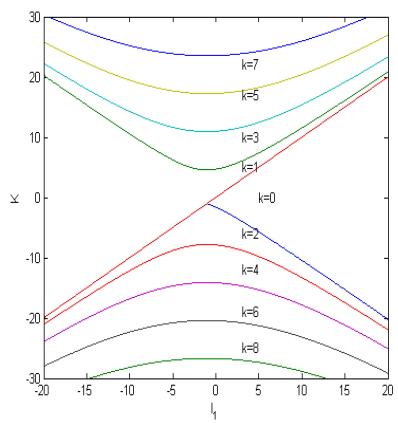

Figure 2. Stability region for the system (2) where $\tau=F_{1}=l_{2}=1$.

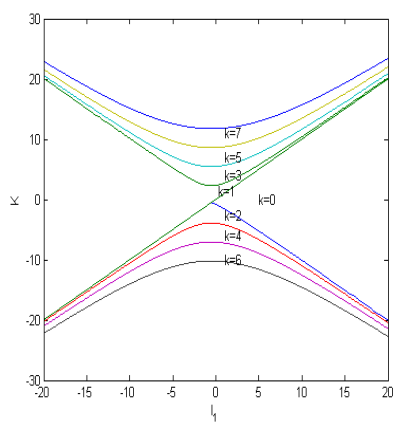

Figure 3. Stability region for the system (2) where $\tau=2$ and $F_{1}=l_{2}=1$.

Hence the asymptotic stable region is the region where $k=0$. By considering these figures, we observed that the asymptotic stability region depends on the delay term. The asymptotic stability region getting smaller as the delay term increases. 
2.5. Theorem. The solution of system (2.2) is asymptotically stable if and only if

$l_{1}>0$ and $\frac{l_{1}}{F_{1}}>K>\frac{-\theta}{\tau \sin (\theta) F_{1}}$. Here $\theta$ values are the roots of $l_{1}-\frac{-\theta \cos (\theta)}{\tau \sin (\theta)}=0$ for $\theta \in(0, \pi)$.

Proof. The asymptotic stability region is given by means of $C_{*}\left(F_{1}, l_{2}\right)$, and (2.9). This region is bounded below by $C_{0}\left(F_{1}, l_{2}\right)$ and above by $C_{*}\left(F_{1}, l_{2}\right)$.

2.2. Special case : Real roots. In this section we consider a specific case in which all characteristic roots are real numbers. Taking the roots $\lambda=\mu \in R$ in (2.4) we have the following

$$
l_{1}=\frac{-\mu\left(\mu+l_{2}\right)+\left(\mu+l_{2}\right) e^{-\mu \tau} F_{1} K}{\left(\mu+l_{2}\right)}
$$

From the model we considered we know that $l_{1}>0$. Hence

$$
l_{1}=-\mu+e^{-\mu \tau} F_{1} K>0
$$

For the asymptotic stability of the system, we give the following theorem.

2.6. Theorem. Consider $l_{1}=-\mu+e^{-\mu \tau} F_{1} K>0$. If $F_{1} K<0$ then the system is asymptotically stable.

Proof. Considering $l_{1}=-\mu+e^{-\mu \tau} F_{1} K>0$ and $F_{1} K<0$ then $\mu \in\left(-\infty, F_{1} K\right)$ which implies that the system is asymptotically stable.

\section{Conclusion}

In this study, we consider the neural field model for two neural population and we investigate the stability of this model by using the D-curves and Stépán's formula. The novelty of this study is to make the stability analysis by getting the characteristic equation of the linearized model. We determine the conditons for the solutions to be asymptotically stable. The asymptotic stability regions are shown on the graphs. As in the stability analysis of the Hayes equation, the asymptotic stability region of the model in this research is bounded by some D-curves namely the singular line and the first D-curve. Moreover as a special case we consider the real roots of the characteristic equation and determine the stability condition. As an open problem, fractional derivatives can be included in the model to make it more realistic and the stability of the system can be analyzed.

\section{References}

[1] Amari, SI. Dynamics of pattern formation in lateral-inhibition type neural fields, Biol. Cybern. 27 (2), 77-87, 1977.

[2] Erman, S., Demir, A. An analysis on the stability of a state dependent delay differential equation, Open Math., 14 (1), 425-435,2016.

[3] Faye G. Symmetry breaking and pattern formation in some neural field equations, PhD thesis, University of Nice- Sophia Antipolis, 2012.

[4] Faye, G., Faugeras, O. Some theoretical and numerical results for delayed neural field equations, Physica D, 239 (9), 561-578, 2010.

[5] Huang, C., Vandewalle, S. An analysis of delay dependent stability for ordinary and partial differential equations with fixed and distributed delays, SIAM J. Sci. Comput., 25 (5), 1608$1632,2004$. 
[6] Insperger, T., Stépán, G. Semi-discretization for time-delay systems, Stability and engineering applications, New York, Springer, 2011.

[7] McCormick, DA., Connors, BW., Lighthall, JW., Prince, DA. Comparative electrophysiology of pyramidal and sparsely spiny stellate neurons of the neocortex, J Neurophysiol, 54 (4), 782-806, 1985.

[8] Stépán, G. Retarded dynamical systems: stability and characteristic functions, England, Longman Scientific \& Technical, 1989.

[9] Veltz, R. Interplay between synaptic delays and propagation delays in neural field equations. Siam J Appl Dyn Syst, 12 (3), 1566-1612, 2013.

[10] Veltz, R., Faugeras, O. Stability of the stationary solutions of neural field equations with propagation delay. Journal of Mathematical Neuroscience 1:1, 2011.

[11] Veltz, R., Faugeras, O. A center manifold result for delayed neural fields equations, Siam J Math Anal 45 (3), 1527-1562, 2013.

[12] Wilson, H., Cowan, J. A Mathematical theory of the functional dynamics of cortical and thalamic nervous tissue. Biol. Cybern. 13 (2), 55-80, 1973. 